\title{
Reduction of blood pressure by aronia berries through inhibition of angiotensin-converting enzyme activity in the spontaneously hypertensive rat kidney
}

\author{
Takuya Yamane ${ }^{a, e, f}$, Miyuki Kozukab, Momoko Imai ${ }^{\text {f }}$, Yoshio \\ Yamamotoc, Iwao Ohkubod, Tatsuji Sakamoto $^{\mathrm{e}}$, Takenori Nakagaki and \\ Yoshihisa Nakanof
}

${ }^{a}$ Institute of Food Sciences, Nakagaki Consulting Engineer and Co., Ltd, Nishi-ku, Sakai 593-8328, Japan; bepartment of Health and Nutrition, Faculty of Human Science, Hokkaido Bunkyo University, Eniwa 061-1449, Japan; 'Department of Ecology and Molecular Biology, Mie University, Iga, Mie 518-0131, Japan; dDepartment of Nutrition, School of Nursing and Nutrition, Tenshi College, Higashi-ku, Sapporo 065-0013, Japan; ${ }^{e}$ Department of Applied Life Sciences, Graduate School of Life and Environmental Sciences, Osaka Prefecture University, Sakai, Osaka 5998531 , Japan; ${ }^{\mathrm{f}}$ Center for Research and Development Bioresources, Research Organization for University-Community Collaborations, Osaka Prefecture University, Sakai, Osaka 599-8570, Japan

*Corresponding author: Takuya Yamane, Center for Research and Development Bioresources, Research Organization for UniversityCommunity Collaborations, Osaka Prefecture University, Sakai, Osaka 5998570 , Japan

Submission Date: April 1st, 2017, Acceptance Date: April 20th, 2017, Publication Date: April 30th 2017

Citation: Yamane T., Kozuka M., Imai M., Yamamoto Y., Ohkubo I., Sakamoto T., Nakagaki T., Nakano Y. Reduction of blood pressure by aronia berries through inhibition of angiotensin-converting enzyme activity in the spontaneously hypertensive rat kidney. Functional Foods in Health and Disease 2017;7(4);280-290

\section{ABSTRACT}

Background: Aronia berries contain many important effects on potential health, with previous studies suggesting that aronia juice is useful for the 
treatment of hypertension.

Objective: To examine whether aronia berries have improvement effects on hypertension through the inhibition of angiotensin-converting enzyme (ACE) activity.

Method: The normal diet containing $10 \%$ freeze-dried aronia berries was administered to five spontaneously hypertensive rats (SHRs) in each group for 28 days, with their body weight, food intake, and systolic blood pressure being measured. At 28 days after administration of aronia berry-containing diets, the serum, lungs, and kidneys were isolated and used for measurement of ACE activity.

Results: In this study, we found that blood pressure in SHRs fed freeze-dried aronia berries-containing diet decreased compared with the blood pressure in SHRs fed normal diet. We also discovered that the catalytic activity of ACE was reduced in the kidney of SHRs fed freeze-dried aronia berriescontaining diet but not in the lungs of aronia berries-administered rats.

Conclusion: Aronia berries have beneficial effects on the amelioration of hypertension through inhibition of the kidney renin-angiotensin system.

Key word: aronia, hypertension improvement, kidney renin-angiotensin system, ACE.

\section{INTRODUCTION}

Aronia berries have been used as the traditional medicine in Russia and Eastern European countries [1]. Aronia berries also have many important potential health effects, including hepatoprotective, cardioprotective, and antidiabetes effects [2]. Aronia juice has been shown to possess beneficial effects on hypertension [3-5]. We have recently discovered that aronia juice inhibits dipeptidyl peptidase IV (DPP IV) activity, which degrades glucose insulinotropic peptide and glucagon-like peptide-1 [6], leading to the beneficial effect of aronia juice on the improvement of diabetes through inhibition of DPP IV and $\alpha$-glucosidase activities [7].

In this study, we found that the blood pressure in SHRs fed freeze-dried aronia berries-containing diet was decreased compared with blood pressure in SHRs fed normal diet. We also discovered that the catalytic activity of angiotensin-converting enzyme (ACE) was reduced in the kidney of SHRs fed a freeze-dried aronia berries-containing diet. Interestingly, inhibition of 
ACE activity was not observed in the lung and serum of fed rats. These results indicate that aronia berries have effects on the mitigation of hypertension through the kidney renin-angiotensin system.

\section{MATERIALS AND METHODS}

\section{Materials}

Aronia berries were obtained from Hokusan (Kitahiroshima, Japan), and they were freeze-dried. Aronia juice was obtained from Nakagaki Consulting Engineer (Osaka, Japan). ACE was purified from porcine seminal plasma [8]. Briefly, $500 \mathrm{ml}$ of porcine seminal plasma was centrifuged and then the supernatant was collected and dialyzed against $20 \mathrm{mM}$ Tris- $\mathrm{HCl}$ buffer $(\mathrm{pH}$ $8.0)$ at $4{ }^{\circ} \mathrm{C}$ for 8 hours. The dialyzed solution was applied to an UNO sphere Q column chromatography and fractionated. The ACE activity of each fraction was measured and fractions containing high ACE activity were collected and dialyzed. Collected fractions were then applied to the UNO sphere Q column chromatography, and fractions with high ACE activity were used for measurement of their proteolytic activity. Z-Lys-Ala-Met-MCA was purchased from Peptide Institute (Osaka, Japan). All other chemicals were of analytical grade and purchased from Wako Pure Chemicals (Osaka, Japan).

\section{Animals}

SHRs were obtained from Japan SLC (Tokyo, Japan). All rats were housed under the SPF condition and all of the rats were basically fed with normal diet (CRF-1, Oriental Yeast, Inc. Tokyo, Japan) for 4 weeks. Male rats at 8 weeks of age were divided into two groups, a group fed a normal diet (Control group) and a group fed a normal diet containing 10\% freeze-dried aronia berries (Aronia group). One-hundred g of aronia berries corresponds to $47 \mathrm{kcal}$. To examine the improvement effect of aronia berries on hypertension, the normal diet containing $10 \%$ freeze-dried aronia berries was administered to five rats in each group for 28 days, and their body weight and food intake were also measured. At 28 days after administration of aronia berry-containing diets, serum, lungs, and kidneys were isolated and used for later experiments.

\section{Blood pressure measurement}

Systolic blood pressure was measured using MK-2000 sphygmomanometer (Muromachi kikai, Tokyo, Japan). 
Measurement of ACE activity was described previously [8]. Briefly, the enzyme activity of ACE was examined by measuring the fluorometrical number (excitation, $380 \mathrm{~nm}$; emission, $440 \mathrm{~nm}$ ) of liberation of 7-amino-4methylcoumarin (AMC) in a mixture containing $10 \mu 1$ of $10 \mathrm{mM} \mathrm{Z}$-Lys-AlaMet-MCA, $100 \mu 1$ of $0.5 \mathrm{M}$ Tris-HCl buffer $(\mathrm{pH} 7.5), 5 \mu 1$ of 1 M 2mercaptoethanol, $20 \mu 1$ of serum, lung and kidney lysates, and water $(18 \mathrm{~m} \Omega)$ in a total volume of $1 \mathrm{ml}$. After incubation of the mixture at $37^{\circ} \mathrm{C}$ for $30 \mathrm{~min}$, $2 \mathrm{ml}$ of $0.2 \mathrm{M}$ acetic acid was added to the mixture to stop the reaction. Fluorescence intensity of AMC was measured using an F-2500 fluorescence spectrophotometer (Hitachi, Japan). Enzyme assays were also carried out using $100 \mu \mathrm{g}$ of total protein from each tissue. The enzyme concentration was quantified using fluorescence intensity of AMC using the standard curve of $\mathrm{AMC}$ concentrations against fluorescence intensity. One unit of activity was defined as the amount of enzyme that hydrolyzed $1 \mu$ mol of the substrate per min.

\section{Preparation of aronia water extract and juice}

Aronia berries were washed and then their seed and skin were removed from aronia berries. One-hundred g of aronia berries without seed and skin was homogenated using a mixer and centrifuged. The supernatant was collected and used as an aronia water extract. Raw juice, on the other hand, was obtained by cold press, percolated, and pasteurized.

\section{In vitro ACE inhibitory assay}

ACE inhibitory activity was measured using the fluorometrical number (excitation, $380 \mathrm{~nm}$; emission, 440nm) of liberation of AMC. Reaction mixture containing $100 \mu 1$ of $0.5 \mathrm{M}$ Tris-HCl buffer $(\mathrm{pH} 7.5), 5 \mu 1$ of $1 \mathrm{M} 2$ mercaptoethanol, $20 \mu 1$ of purified ACE solution (32 mUnits), and water $(18 \mathrm{~m} \Omega)$ in a total volume of $990 \mu 1$ was incubated at room temperature for $10 \mathrm{~min}$. Afterwards, $10 \mu 1$ of $10 \mathrm{mM} \mathrm{Z}$-Lys-Ala-Met-MCA was added to the mixture and incubated at $37^{\circ} \mathrm{C}$ for $30 \mathrm{~min}$. Two $\mathrm{ml}$ of $0.2 \mathrm{M}$ acetic acid was then added to the mixture to stop the reaction. Fluorescence intensity of AMC was measured using an F-2500 fluorescence spectrophotometer 
(Hitachi, Japan). One unit of activity was defined as the amount of enzyme that hydrolyzed $1 \mu$ mol of the substrate per min.

\section{Statistical analysis}

Data are expressed as means \pm S.E. Statistical analyses were performed using analysis of variance (one-way ANOVA) followed by an unpaired Student's ttest. For comparison of multiple samples, the Dunnett test was used.

\section{Ethics statement}

All animal experiments were carried out in accordance with the National Institutes of Health Guide for the Care and Use of Laboratory Animals, and the protocols were approved by the Committee for Animal Research at Safety Research Institute for Chemical Compounds (the permit number NP013-517).

\section{RESULTS}

Body weight and food intake As shown in Fig. 1, the body weight was not significantly altered between the control group and aronia group. Food intake was also not significantly altered between the control group and aronia group (Fig. 2A). As shown in Fig. 2B, aronia intake was constant in aronia berries-administered SHRs for 28 days.

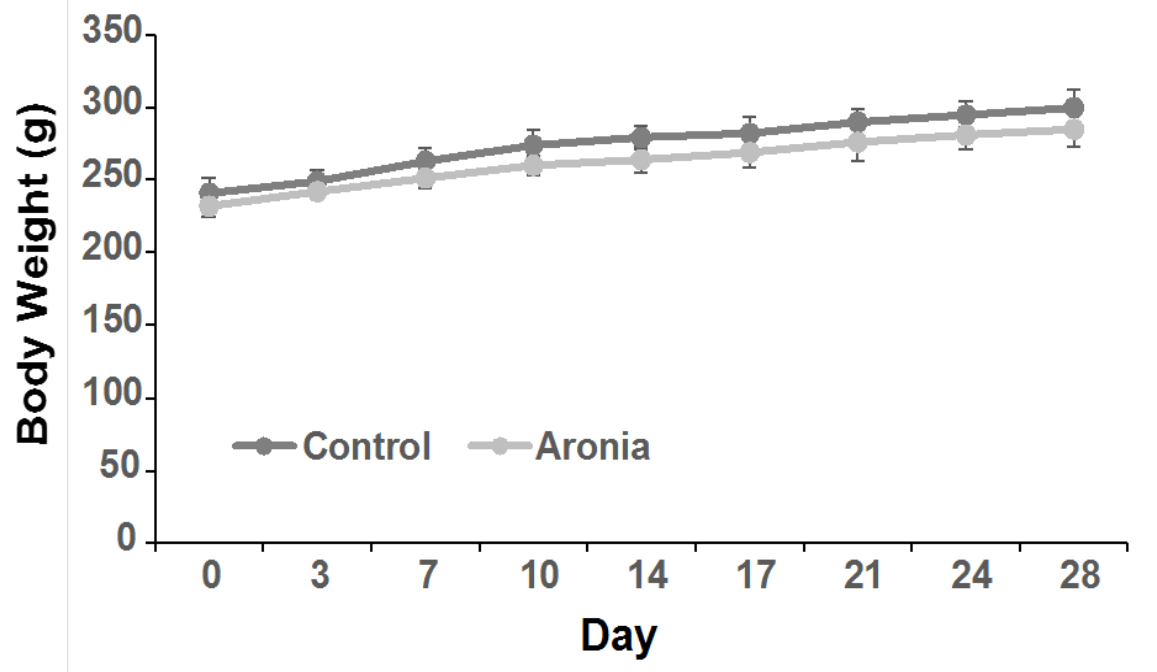

Body Weught $(\mathrm{g})$

\begin{tabular}{llllllllll} 
Day & 0 & 3 & 7 & 10 & 14 & 17 & 21 & 24 & 28 \\
\hline
\end{tabular}

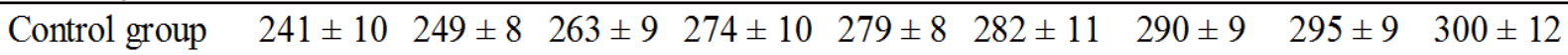

\begin{tabular}{llllllllll} 
Aronia group & $232 \pm 7$ & $242 \pm 5$ & $251 \pm 7$ & $260 \pm 7$ & $264 \pm 9$ & $269 \pm 10$ & $276 \pm 13$ & $281 \pm 10$ & $285 \pm 12$ \\
\hline
\end{tabular}


Figure 1. Differences in body weight between control and aronia berriesadministered SHRs. Body weight was measured for 28 days, and the results did not reveal a significant difference between the control and aronia groups. $\mathrm{n}=5$.

\section{A. Food intake}

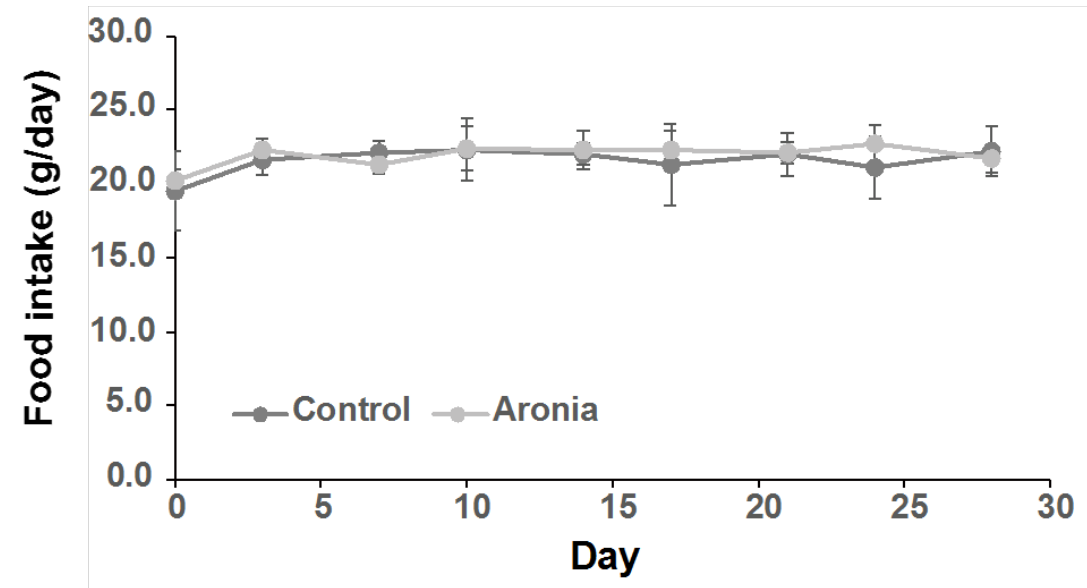

\begin{tabular}{cccccccccc}
\hline \multicolumn{10}{c}{ Food intake (g/day) } \\
\hline Day & 0 & 3 & 7 & 10 & 14 & 17 & 21 & 24 & 28 \\
\hline Control group & $19.5 \pm 2.7$ & $21.6 \pm 1.0$ & $22.1 \pm 0.8$ & $22.3 \pm 2.1$ & $22 \pm 0.7$ & $21.3 \pm 2.8$ & $22 \pm 1.5$ & $21.1 \pm 2.1$ & $22.2 \pm 1.7$ \\
Aronia group & $20.2 \pm 0.8$ & $22.3 \pm 0.8$ & $21.3 \pm 0.6$ & $22.4 \pm 1.5$ & $22.3 \pm 1.3$ & $22.3 \pm 1.3$ & $22.1 \pm 0.7$ & $22.7 \pm 1.3$ & $21.7 \pm 0.9$ \\
\hline
\end{tabular}

\section{B. Aronia intake}

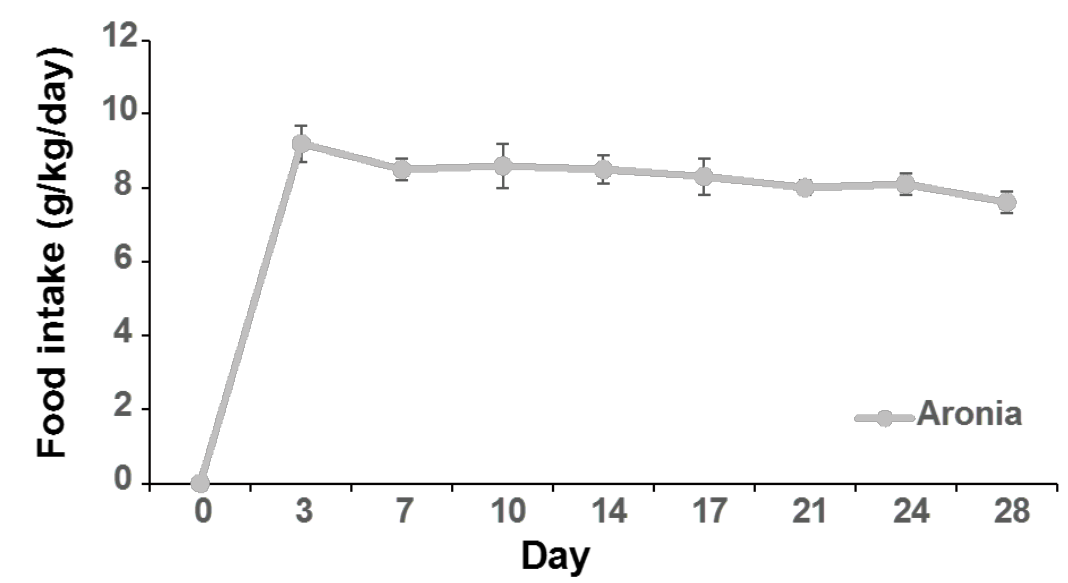

\begin{tabular}{ccccccccccc}
\hline \multicolumn{10}{c}{ Aronia intake $(\mathrm{g} / \mathrm{kg} /$ day) } \\
\hline Day & 0 & 3 & 7 & 10 & 14 & 17 & 21 & 24 \\
\hline Control group & 0.0 & 0.0 & 0.0 & 0.0 & 0.0 & 0.0 & 0.0 & 0.0 & 0.0 \\
Aronia group & 0.0 & $9.2 \pm 0.5$ & $8.5 \pm 0.3$ & $8.6 \pm 0.6$ & $8.5 \pm 0.4$ & $8.3 \pm 0.5$ & $8.0 \pm 0.2$ & $8.1 \pm 0.3$ & $7.6 \pm 0.3$ \\
\hline
\end{tabular}

Figure 2. Differences in food intake between control and aronia berriesadministered SHRs. A. Food intake was measured for 28 days, and the results did not show the significant difference between control and aronia groups. $\mathrm{n}=5$. B. Aronia intake was measured for 28 days. $\mathrm{n}=5$. 
Beneficial effect of aronia berries on hypertension in SHRs

To examine the reduction of blood pressure on SHRs administered aronia berries, blood pressure was measured at every 3 or 4 days for 28 days. As shown in Fig. 3, the blood pressure of SHRs administered aronia berries was lower than that of SHRs without aronia berries from day 3 to 28. Significant reduction of blood pressure of aronia berries-administered rats was observed at day 28 .

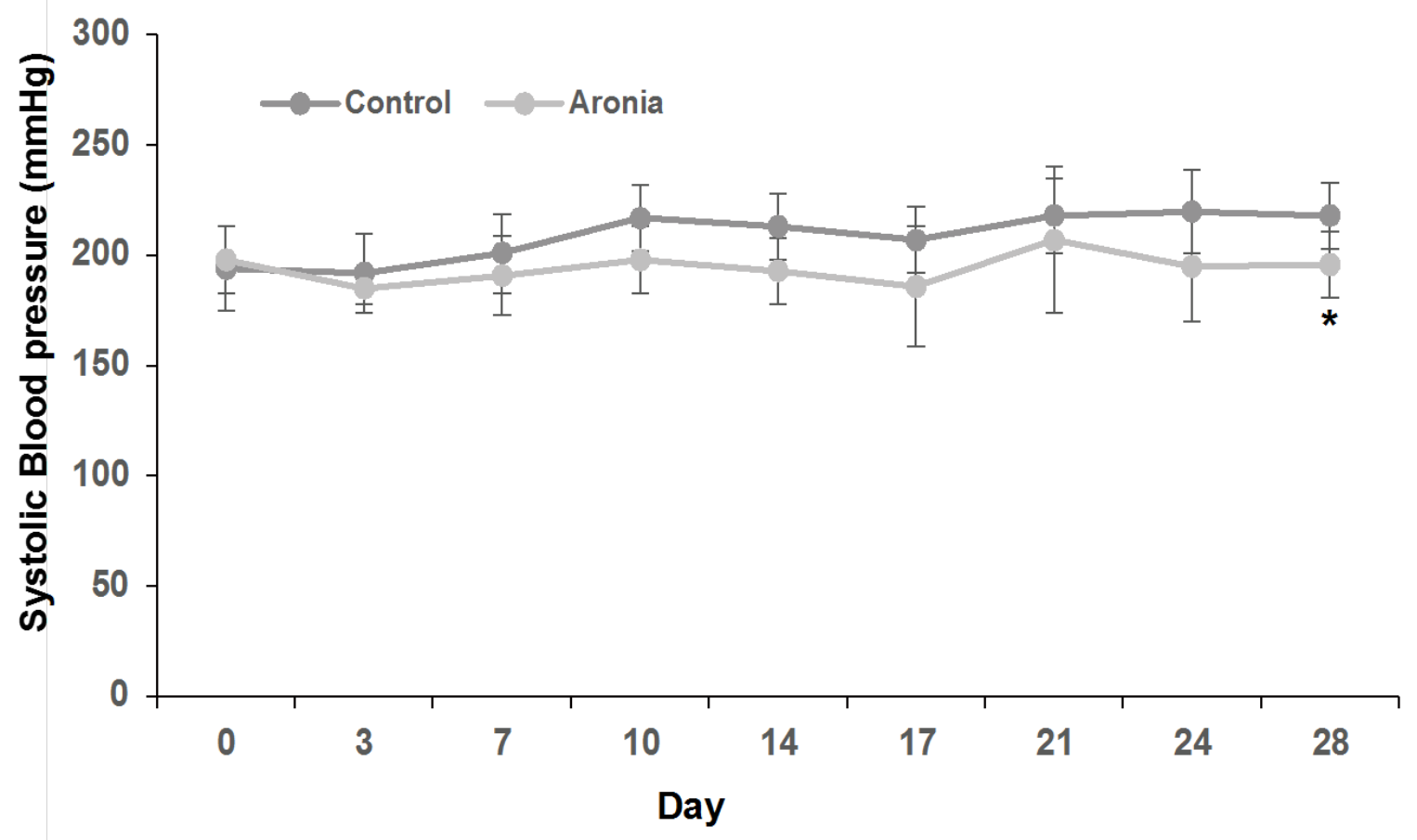

\begin{tabular}{cccccc|c|c|c|c|c|c|}
\hline \multicolumn{10}{c}{ Systolic blood pressure (mmHg) } \\
\hline \multicolumn{1}{c}{ Day } & 0 & 3 & 7 & 10 & 14 & 17 & 21 & 24 & 28 \\
\hline Control group & $194 \pm 19$ & $192 \pm 18$ & $201 \pm 18$ & $217 \pm 15$ & $213 \pm 15$ & $207 \pm 15$ & $218 \pm 17$ & $220 \pm 19$ & $218 \pm 15$ \\
\cline { 2 - 11 } Aronia group & $198 \pm 15$ & $185 \pm 7$ & $191 \pm 18$ & $198 \pm 15$ & $193 \pm 15$ & $186 \pm 27$ & $207 \pm 33$ & $195 \pm 25$ & $196 \pm 15^{*}$ \\
\hline
\end{tabular}

Figure 3. Differences in blood pressure between control and aronia berriesadministered SHRs. SHRs were administered a diet containing $10 \%$ freezedried aronia berries for 28 days and their blood pressure was measured at every 3 or 4 days for 28 days. There were significant differences of blood pressure between the control and aronia berries- administered SHRs. ${ }^{*} p<0.05$, $* * p<0.01, \mathrm{n}=5$.

\section{Inhibitory effect of aronia berries on ACE activity in SHRs}

Twenty-eight days after administration of aronia berries to SHRs, serum, lungs, and kidneys were extracted from rats, and their ACE activities were measured. As shown in Fig. 4A, serum ACE activities in aronia berriesadministered SHRs were not significantly altered compared to those in SHRs administered normal diet. On the other hand, Lung ACE activities in aronia 
berries-administered SHRs were significantly increased compared to those in normal diet-administered SHRs (Fig. 4B). However, kidney ACE activities in aronia berries-administered SHRs were significantly reduced compared to those in normal diet-administered SHRs (Fig. 4C).

A. Serum

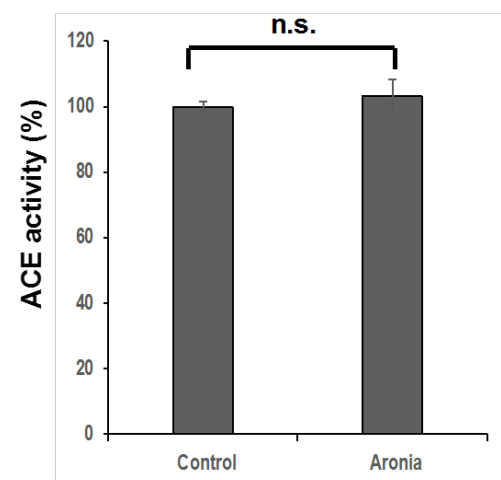

B. Lung

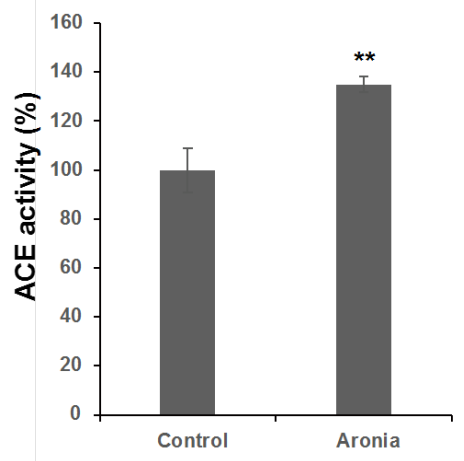

C. Kidney

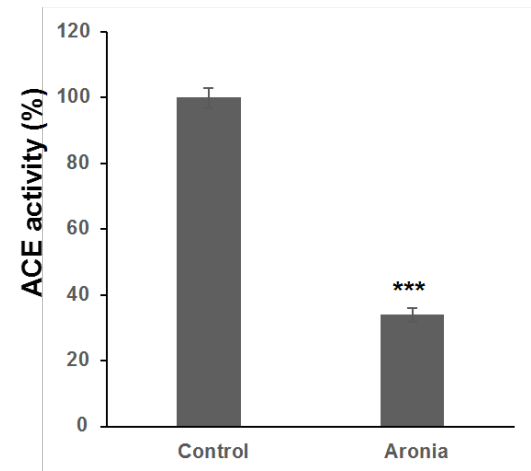

Figure 4. ACE activities in serum, lungs, and kidneys of berriesadministered SHRs. Aronia berries were administered orally to rats. After 28 days, serum, lungs, and kidneys were obtained and their ACE activities were measured. A. Serum, B. Lung, C. Kidney. ${ }^{* *} p<0.01,{ }^{* * *}<<0.001$, n.s.: not significant. $\mathrm{n}=5$.

Inhibitory effect of aronia water extract and juice on ACE activity To examine the inhibitory effect of aronia water extract and juice on ACE activity, ACE activity was measured using purified ACE from porcine seminal plasma and a synthetic substrate, Z-Lys-Ala-Met-MCA, in vitro. As shown in Figs. 5A and B, inhibition of ACE activity was observed after reactions with aronia water extract and juice in a dose-dependent manner and ACE activities in reactions with extract and $30 \mu 1$ of juice were reduced to about $7 \%$ and $8 \%$ respectively, of those in the vehicle control. Amounts of total anthocyanin present in aronia juice were also shown. 
A. Extract

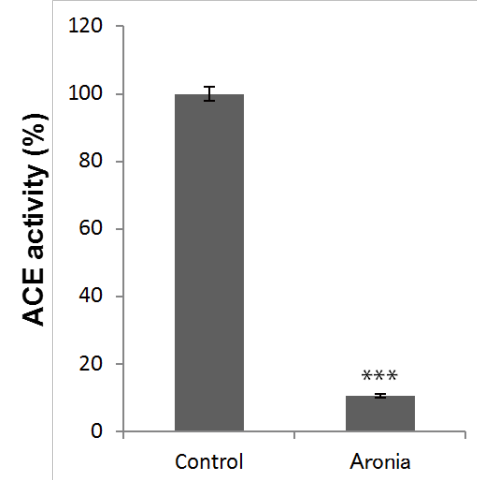

B. Juice

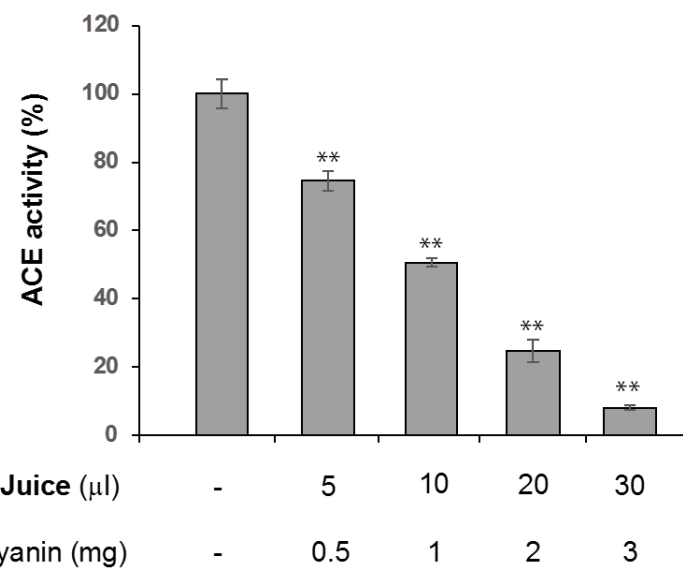

Figure 5. Inhibitory effect of aronia berries and juice on ACE activity. A. Purified ACE was reacted with aronia berries water extract, and ACE activity was measured as described in Materials and methods.

B. Purified ACE was reacted with various amounts of aronia juice and their ACE activity was measured as described in Materials and methods. Amounts of total anthocyanin present in aronia juice were also shown. The method for measurement of total anthocyanin was previously described [15]. $* * p<0.01, * * * p<0.001, \mathrm{n}=5$.

\section{DISCUSSION}

We discovered that blood pressure was reduced in SHRs that had been administered aronia berries. Previous studies showed that hypertension is reduced in aronia berries-administered human or mice [3, 4]. These previous studies and our findings indicate that aronia berries have the beneficial effect on the amelioration of hypertension. Since the body weight and food intake were not different between the control group and aronia group, these results support that the reduction of blood pressure is induced by component(s) in aronia berries. Furthermore, we found that ACE activity was reduced in the kidney, but not the lungs and serum of SHRs that had been administered aronia berries. We also found that aronia water extract and aronia juice possessed inhibitory activity against ACE. Perrsson et al. have reported that anthocyanidins such as cyanidin, delphindin, and malvidin inhibit ACE activity [9], and Guerrero et al have recently reported that ACE activity is inhibited by flavonoids including luteolin, quercetin, and quercetin 3-O-glycoside [10]. Furthermore, Hibiscus sabdariffa, anthocyanins, and flavonoids such as cyanidin and quercetin 3-O-glycoside possess ACE inhibitory activity [11]. Anthocyanins and flavonoids, including cyanidin, cyanidin 3-O-glycoside, and quercetin 3-O-glycoside are 
present in aronia berries [12]. Our findings and previous studies indicate that ACE activity is inhibited by cyanidin, cyanidin 3-O-glycoside, and quercetin 3-O-glycoside present in aronia berries. The presence of renin, angiotensinogen, $\mathrm{ACE}$, and angiotensin II type 1 receptors within renal tissues stimulates the local renin-angiotensin system and angiotensin II locally generated by renal ACE is a master regulator of the nephron sodium transport and plays a key role in experimental hypertension [13]. Since components from aronia berries inhibit renal ACE activity, it is thought that the beneficial effect of aronia berries on hypertension is caused by the inhibition of the local renin-angiotensin system in the SHR kidney. However, a previous human study revealed that reduction of ACE activity was observed in plasma from patients that had been administered aronia melanocarpa Elliot extract [14]. These studies suggest aronia berries may possess different mechanisms between human beings and rat in terms of the inhibitory effect against ACE. Furthermore, the facts that aronia berries increase ACE activity in the lung of SHRs, that anthocyanins and/or flavonoids present in aronia berries inhibit ACE activity in the kidney, and that the reduction of ACE activity in the local renin-angiotensin system contributes to reduction of hypertension which thereby gives rise to the possibility that aronia berries become a useful drug for prevention of hypotension.

\section{CONCLUSIONS}

In conclusion, aronia berries inhibit $A C E$ activity in the kidney and reduction of ACE activity in the local renin-angiotensin system. Aronia berries may become a useful drug for the prevention of hypotension.

List of Abbreviation: ACE, angiotensin-converting enzyme; SHR, spontaneously hypertensive rat; AMC, 7-amino-4-methylcoumarin.

Conflict of Interest: The authors have declared that no competing interests exist.

Author's Contributions: All authors contributed to this study.

\section{REFERENCES}

1. Kokotkiewicz A, Jaremicz Z, Luczkiewicz M: Aronia plants: a review of traditional use, biological activities, and perspectives for modern medicine. J Med Food 2010, 13: 255-269.

2. Kulling ES, Rawel MH: Chokeberry (Aronia melanocarpa) -A review on the characteristic components and potential health effects. Planta Med 2008, 74: 1625-1634. 
3. Tjelle TE, Holtung L, Bøhn SK, Aaby K, Thoresen M, Wiik SA, Paur I, Karlsen AS, Retterstøl K, Iversen PO, Blomhoff R: Polyphenol-rich juices reduce blood pressure measures in a randomised controlled trial in high normal and hypertensive volunteers. Br J Nutr 2015, 114: $1054-1063$.

4. Ciocoiu M, Badescu L, Miron A, Badescu M: The involvement of a polyphenol-rich extract of black chokeberry in oxidative stress on experimental arterial hypertension. Evid Based Complement Alternat Med 2013, 2013: 912769.

5. Broncel M, Koziróg-Kołacińska M, Andryskowski G, Duchnowicz P, Koter-Michalak M, Owczarczyk A, Chojnowska-Jezierska J: Effect of anthocyanins from Aronia melanocarpa on blood pressure, concentration of endothelin-1 and lipids in patients with metabolic syndrome. Pol Merkur Lekarski 2007, 23: 116-119.

6. Kozuka M, Yamane T, Nakano Y, Nakagaki T, Ohkubo I, Ariga H: Identification and characterization of a dipeptidyl peptidase IV inhibitor from aronia juice. Biochem Biophys Res Commun 2015, 465: $433-436$.

7. Yamane T, Kozuka M, Konda D, Nakano Y, Nakagaki T, Ohkubo I, Ariga H: Improvement of blood glucose levels and obesity in mice given aronia juice by inhibition of dipeptidyl peptidase IV and $\alpha$ glucosidase. J Nutr Biochem 2016,31: 106-112.

8. Takeuchi K, Araki H, Sakaue T, Yamamoto Y, Fujiwara M, Nishi K, Ohkubo I: Porcine germinal angiotensin I-converting enzyme: isolation, characterization and molecular cloning. Comp Biochem Physiol B Biochem Mol Biol 2007, 146: 215-226.

9. Persson IA, Persson K, Andersson RG: Effect of Vaccinium myrtillus and its polyphenols on angiotensin-converting enzyme activity in human endothelial cells. J Agric Food Chem 2009, 57: 4626-4629.

10. Guerrero L, Castillo J, Quiñones M, Garcia-Vallvé S, Arola L, Pujadas G, Muguerza B: Inhibition of angiotensin-converting enzyme activity by flavonoids: structure-activity relationship studies. PLoS One 2012, 7: e 49493.

11.Da-Costa-Rocha I, Bonnlaender B, Sievers H, Pischel I, Heinrich M: Hibiscus sabdariffa L. - a phytochemical and pharmacological review. Food Chem 2014, 165: 424-443.

12. Skoczyñska A, Jêdrychowska I, Porêba R, AffelskaJercha A, Turczyn B, Wojakowska A, Andrzejak R: Influence of chokeberry juice on arterial blood pressure and lipid parameters in men with mild hypercholesterolemia. Pharm Rep 2007, 59: 177-182.

13.Bernstein KE, Giani JF, Shen XZ, Gonzalez-Villalobos RA: Renal angiotensin-converting enzyme and blood pressure control. Curr Opin Nephrol Hypertens 2014, 23: 106-112.

14. Sikora J, Broncel M, Mikiciuk-Olasik E: Aronia melanocarpa Elliot 
reduces the activity of angiotensin I-converting enzyme-in vitro and ex vivo studies. Oxid Med Cell Longev 2014, 2014: 739721.

15. Cassinese C, de Combarieu E, Falzoni M, Fuzzati N, Pace R, Sardone $\mathrm{N}$ : New liquid chromatography method with ultraviolet detection for analysis of anthocyanins and anthocyanidins in Vaccinium myrtillus fruit dry extracts and commercial preparations. J AOAC Int 2007, 90: 911-919. 